\title{
ChAdOx1 nCoV-19 vaccine and its self-reported adverse events: a cross-sectional study from Western Nepal
}

Arun Gautam', Nixon Dangol ${ }^{2}$, Urza Bhattarai ${ }^{3}$, Sandesh Paudel ${ }^{2}$, Bipin Poudel ${ }^{4}$, Shankar Gautam ${ }^{5}$, Prabhat Sharma ${ }^{6}$ Surendra Uranw', Sanjib K Sharma ${ }^{1}$

1 Department of Internal Medicine, BP Koirala Institute of Health Sciences, Dharan, Nepal, 2 Galkot Primary Health Care Center, Baglung, Nepal, 3 Department of Geriatric Medicine, All India Institute of Medical Sciences, New Delhi, India, ${ }^{4}$ Nepal College of Management, Kathmandu, Nepal, 5 National Institute of Ayurveda, Jaipur, India, ${ }^{6}$ Shankarapur Hospital, Kathmandu, Nepal

Keywords: aefi, chadox1 ncov-19 vaccine, adverse events, vaccine, covid-19

https://doi.org/10.29392/001c.25471

Journal of Global Health Reports

Vol. 5, 2021

\section{Background}

There are various COVID-19 vaccines launched in different parts of the world. As the vaccination drive is increasing, the reports of adverse events following immunization (AEFI) are increasingly reported. Therefore, this research aims to document the adverse events and their determinants following COVID-19 vaccination.

\section{Methods}

This was a cross-sectional study conducted in a primary healthcare setting of Western Nepal. Those individuals who took the first dose of the ChAdOx1 nCoV-19 vaccine in the health care center $(\mathrm{N}=425)$ were eligible for the study. The details to contact the study participants were obtained from the vaccination center. Patients were contacted via phone calls by investigators. Information on demographic details, comorbidities and experiences of any sort of adverse events following vaccination was collected using pre-structured questionnaire. Descriptive statistics was done to describe the categorical variables in frequency and percentage whereas mean, median, standard deviation and interquartile range were calculated for quantitative variables. Binary logistic regression was carried out to explore the potential determinants of AEFI.

\section{Results}

A total of 302 (71.05\%) people were randomly selected from 425 targeted people for vaccination at the primary health care center. A total of 276 (91.3\%) people vaccinated with ChAdOx1 nCoV-19 vaccine responded to telephone call. Mean age of participants was $33.63( \pm 11.14$ ) years and majority of them were males $(54.74 \%) .91 .6 \%$ of participants developed some form of AEFI. Among them, 46.6\% developed mild AEFI, and 53.4\% developed moderate AEFI. Pain on the injection site, generalized weakness, fever, headache, joint and muscle pain, dizziness, and loss of appetite are found to be the "Very common" adverse events. Females tend to develop moderate AEFI than males (OR 2.369; 95\% confidence interval, $\mathrm{CI}=1.398-4.014 ; P=0.001)$. Age did not seem to be the determinant of AEFI (OR 0.977; $\mathrm{CI}=0.954-1.002 ; P=0.067)$.

\section{Conclusions}

Adverse events following COVID-19 immunization are common but not severe. The severity of AEFI is more in females compared to males. Age did not come out as a predictor for severity of AEFI. Post-vaccination counseling targeting women will improve the acceptance of the vaccine. We recommend further monitoring of AEFI in larger population.

The COVID-19 pandemic, with its detrimental effects, has overwhelmed health systems and economy throughout the world. Various COVID-19 vaccines have been launched in different parts of the world. ${ }^{1}$ An Adverse events following immunization (AEFI) is defined as "Any untoward medical occurrence which follows immunization and which does not necessarily have a causal relationship with the usage of the vaccine". ${ }^{2}$ Nepal approved the emergency use of the ChAdOx1 nCoV-19 vaccine (COVISHIELD) made by the Serum Institute of India on January 15, 2021. The first drive of vaccination started in Nepal with this vaccine on January $27,2021 .^{3}$ To augment the inoculation initiative, the Min- 
istry of Health and Population, Nepal expanded the vaccination campaign in different levels of health foci including primary health care centers under the supervision of acute AEFI emergency management team to address possible adverse events. The first drive of vaccination's target group were health care staff, sanitation staff, health volunteers, and security personnel involved in the management of dead bodies of people infected with the coronavirus. The second drive of the COVID-19 vaccination started in Nepal on February 08, 2021 and ChAdOx1 nCoV-19 vaccine (COVISHIELD) was the only available vaccine against COVID-19 at that time in Nepal. ${ }^{4}$ The target groups of the second drive were administrative officials, bankers, journalists, and those who missed their dose in the first drive. ${ }^{4,5}$ We conducted this research in a primary health care center located in a rural district of Western Nepal. The second drive of the Vaccination campaign took place at our center on February 17 and February 18, 2021. Until February 18, 2021 a total of 273,166 confirmed cases of COVID-19 were reported from Nepal. There were 96 new cases and 671 active cases reported on the same date. ${ }^{6}$ The coverage area of the primary health care center for the second drive of vaccination included Galkot Municipality and Tarakhola rural Municipality of Baglung district, Nepal. This district in the last one month before the vaccination campaign reported 76 new cases of COVID-19. ${ }^{7}$ There were no active cases present in Galkot municipality and Tarakhola rural municipality at the time of vaccination. Phase $2 / 3$ trial of ChAdOx1 nCoV- 19 Vaccine has shown that this vaccine may have some well tolerated adverse events, fatigue, headache, feverishness, and myalgia being the most common systemic adverse events. ${ }^{8}$ As the vaccination drive is increasing, the reports of AEFI are increasingly reported. Various surveys conducted after the vaccination campaign have also shown the similar adverse events to be more common. They have reported the variation of AEFI with different demographic factors like age, gender and probably with a history of COVID-19 infection. ${ }^{9-11}$ There were very few research studies on AEFI with the COVID-19 vaccine. Hesitancy was one of the challenges with the launch of various types of vaccines against COVID-19. ${ }^{3}$ We were lacking with the scientific data of the Nepalese community. As the scientific evidences could help minimize such hesitancy regarding vaccine use, we undertook this research to study the adverse events following the first dosage of COVID-19 vaccination and to find the determinants of its adverse events. This study of adverse events following the first dosage of the COVID-19 vaccine was planned with a vision that the data from this study can be used to compare with adverse events that may follow with other types of vaccines against COVID-19.

\section{METHODS}

Our study was a cross-sectional study among people who took the first dose of the ChAdOx1 nCoV-19 vaccine during the second drive of the vaccination campaign at Galkot primary health care center, located in the western hilly region of in Nepal. As per the information delivered by the District Health office, Baglung, the number of people of the target group for the second drive in our health care center was es- timated to be 502 and the same number of vaccine dosages were allocated. Those individuals who took the first dose of the ChAdOx1 nCoV-19 vaccine in our health care center $(\mathrm{N}=425)$ were eligible for the study. The vaccine response rate of this population was $84.6 \%$. All the vaccine recipients received information about their possible selection in the research and getting phone calls from the doctors asking about their perceived symptoms post vaccination. Every 425 participants were assigned a number ranging from 1 to 425 . Then the random number was generated using Microsoft excel (Microsoft Inc, Seattle WA, USA) with the algorithm $=$ RANDBETWEEN $(\mathrm{x}, \mathrm{y})$, which produced a random digit assigned to each cell. The repeated random digit generated were skipped. 302 people were randomly selected with an estimated $10 \%$ noncontact and dropout rates. We asked the selected participants about their perceived symptoms and their characteristics, including onset, duration, limitation in daily activities and the requirement of medications or hospitalization, history of any underlying chronic illness, history of use of alcohol or smoking, history of allergy, past history of COVID-19, and history of use of any other vaccination within last 1 month as per the pre-structured questionnaire via phone calls. The questions asked were leading questions regarding the symptoms perceived by them. The questionnaire was formulated by medical doctors who were in regular medical practice at the local community level. The doctors involved in formulating the questionnaire conducted the interview. The interview was conducted 3 to 15 days post vaccination. We could not contact 26 people because of wrong numbers and inaccessible phone calls. The basic response rate to the questionnaire was $91.3 \%$. 2 people were excluded from analysis as they were found to have used antipyretics immediately after vaccination with fear of adverse events. A total of 274 participants were analyzed. The sampling scheme is shown in Figure 1. We took the data like vaccination date and time, weight, height and BMI from hospital records. Our study included only those individuals who got the first dose of the COVID-19 vaccine and consented to research participation.

We excluded those vaccine recipients who were unreachable with phone calls, those who could not give an interview in phone calls due to listening or speaking problems, those who were uncooperative and denied complying with the interview process and those participants who took antipyretics as prophylaxis. Also, we excluded those participants who attributed the symptoms to causes other than vaccination.

This study is carried out as per the international conference of harmonization-good clinical practices guidelines (ICH-GCP). An informed written consent was taken from each patient willing to participate before the commencement of the study. The patients were free to withdraw their names from the study at any time without giving any reason.

We defined the post vaccination symptoms in our study as per the definition criteria used in the Phase $2 / 3$ trial of ChAdOx1 nCoV-19 vaccine and Common Terminology Criteria for Adverse Events (CTCAE) criteria by the National Cancer Institute. ${ }^{8,12}$ 


\section{MILD SYMPTOMS}

1. Any symptom with duration less than or equal to 48 hours AND not impairing daily activity AND no use of medications to relieve those symptoms.

2. Vomiting was considered mild if: vomiting frequency of 1 episode in 24 hours AND not impairing daily activities AND no use of medications to relieve those symptoms.

3. Loose motions were considered mild if: Loose stool frequency of fewer than 4 stools a day AND not impairing daily activities AND no use of medications.

\section{MODERATE SYMPTOMS}

1. Duration of more than 48 hours OR Impairment of daily activities OR Need minor medical interventions like oral medications

2. Vomiting was considered moderate if: vomiting frequency of 2-5 episodes in 24 hours OR not impairing daily activities OR use of oral medications to relieve those symptoms.

3. Loose motions were considered moderate if: Loose stool frequency of 4 to 6 stools a day OR impairing daily activities OR use of oral medications.

\section{SEVERE SYMPTOMS}

1. Need of hospitalization and management.

2. Vomiting was considered severe if: vomiting frequency of 6 or more episodes in 24 hours OR need of hospitalization.

3. Loose motions were considered severe if: Loose motion frequency of 7 or more episodes a day OR need of hospitalization.

We defined no, mild, moderate and severe AEFI as follows:

1. No AEFI: Those who had no post vaccination symptoms.

2. Mild AEFI: Those who had at least one mild symptom without any moderate or severe symptoms.

3. Moderate AEFI: Those who had at least one moderate symptom.

4. Severe AEFI: Those who had at least one severe symptom.

After the collection and organization of data, summary measures such as mean, median, standard deviation, frequency and percentage were computed wherever applicable. AEFI severity with two levels (no/mild AEFI and Moderate AEFI) was considered as the outcome variable. Firstly, binary logistic regression on a single individual factor was performed to identify its significance on the dependent variable. Once, the significant factors were identified they were forwarded to multiple regression modelling of the outcome variable. The estimated parameters were interpreted using odds ratio (OR). Finally, the adequacy of the fitted model was assessed via Omnibus test, Nagelkerke R square and Hosmer and Lemeshow Test. All of the statistical tests were performed at $\alpha=0.05$. Statistical analysis of data was done using IBM SPSS Statistics for Windows, Version 23.0.

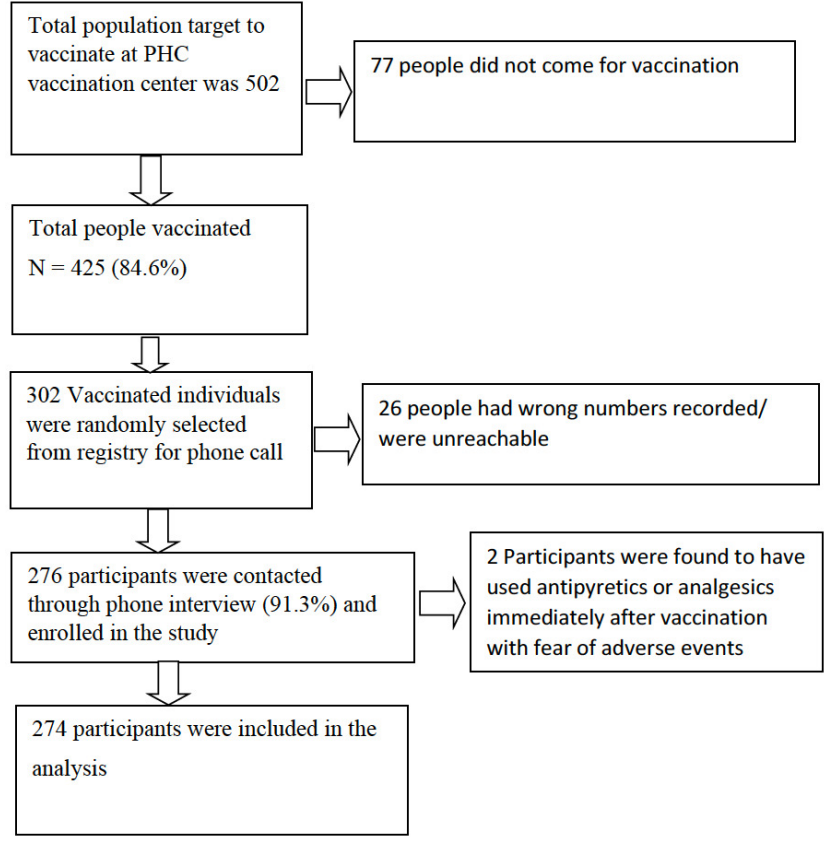

Figure 1. Sampling scheme

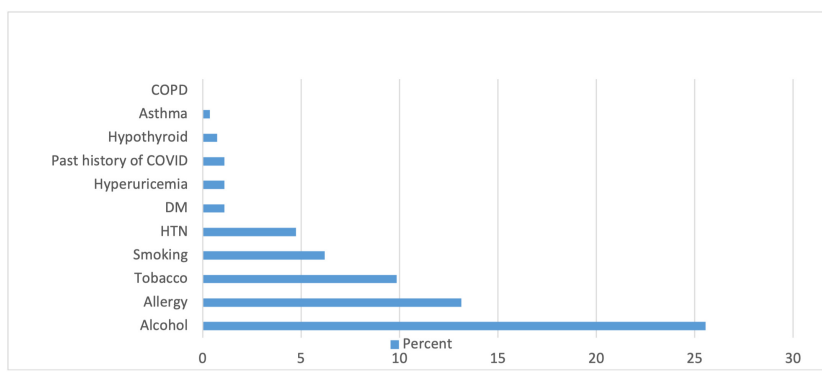

Figure 2. Prevalence of chronic illness, substance abuse and COVID-19 infection in overall Samples

(IBM Corporation, New York, USA).

\section{RESULTS}

Overall, $91.6 \%$ of the participants developed some form of AEFI following vaccination. Among them, 46.6\% developed mild AEFI, 53.4\% developed moderate AEFI. The remaining 8.4\% developed no AEFI. 21.2\% of the participants had impairment of their daily activities following vaccination and $12.8 \%$ of the participants used some form of oral medications to relieve their symptoms. The medications used mainly were analgesics or antipyretics. The baseline characteristics of the data we analyzed to find out the possible determinants of AEFI are shown in Table 1 and Table 2. The prevalence of various underlying chronic illnesses, alcohol use, smoking, tobacco use and minor allergies among the sample is shown in Figure 2.

As per the WHO tool for global use on assessment of adverse events following immunization, the "very common" adverse events are those with a frequency of more than or equal to $10 \% .{ }^{13}$ The frequency of "very common" AEFI, 
Table 1. Overall versus group wise comparison of severity of AEFI with continuous variables.

\begin{tabular}{|l|c|c|c|c|c|c|c|c|c|}
\hline Variables & N & Mean & Median & S.D. & Min. & Max. & Quartiles \\
\hline \multicolumn{7}{|c|}{ Overall participants } \\
\hline Age & 274 & 33.63 & 31 & 11.14 & 18 & 73 & 25 & 40 \\
\hline Weight (in KG) & 274 & 63.19 & 61 & 12.28 & 40 & 100 & 54 & 71 \\
\hline BMI & 274 & 24.56 & 23.68 & 4.7 & 15.45 & 40.36 & 21.52 & 26.89 \\
\hline \multicolumn{7}{|c|}{ No and Mild AEFI } \\
\hline Age & 140 & 35.78 & 33 & 12.27 & 19 & 73 & 26.5 & 44.5 \\
\hline Weight (in KG) & 140 & 64.4 & 64 & 12.13 & 42 & 95 & 55 & 72.5 \\
\hline BMI & 140 & 24.58 & 23.76 & 4.38 & 15.44 & 40.34 & 21.52 & 27.4 \\
\hline \multicolumn{7}{|c|}{} \\
\hline Age & 134 & 31.38 & 29 & 9.35 & 18 & 65 & 24 & 37 \\
\hline Weight (in KG) & 134 & 61.93 & 59.5 & 12.34 & 40 & 100 & 54 & 70 \\
\hline BMI & 134 & 24.53 & 23.68 & 5.03 & 15.71 & 40.36 & 21.35 & 26.69 \\
\hline
\end{tabular}

Table 2. Overall versus group wise comparison of severity of AEFI with gender and ethnicity

\begin{tabular}{|l|l|c|c|c|}
\hline \multicolumn{2}{|c|}{ Variables } & $\begin{array}{c}\text { No and Mild AEFI } \\
\left(\mathrm{n}_{\mathbf{1}}=140\right)\end{array}$ & $\begin{array}{c}\text { Moderate AEFI } \\
\left(\mathrm{n}_{\mathbf{2}}=134\right)\end{array}$ & $\begin{array}{c}\text { Overall } \\
(\mathbf{n}=\mathbf{2 7 4})\end{array}$ \\
\hline \multirow{3}{*}{ Gender } & Male & $67.10 \%$ & $41.80 \%$ & $54.74 \%$ \\
\cline { 2 - 5 } & Female & $32.90 \%$ & $58.20 \%$ & $45.26 \%$ \\
\hline \multirow{3}{*}{ Ethnicity } & Brahmin/Chhetri & $52.90 \%$ & $53 \%$ & $53 \%$ \\
\cline { 2 - 5 } & Others & $47.10 \%$ & $47 \%$ & $47 \%$ \\
\hline
\end{tabular}

Table 3. Summary measures of very common AEFI with COVID-19 vaccine

\begin{tabular}{|l|c|c|}
\multicolumn{1}{|c|}{ AEFI } & Onset (hours) mean \pm SD & $\begin{array}{c}\text { Duration(hour) } \\
\text { Median (IQR) }\end{array}$ \\
\hline Pain on injection site & $9.86 \pm 7.61$ & $48(48)$ \\
\hline Weakness & $12.30 \pm 6.31$ & $24(24)$ \\
\hline Fever & $12.76 \pm 7.16$ & $24(12)$ \\
\hline Headache & $11.41 \pm 6.61$ & $24(15)$ \\
\hline Joint and muscle pain & $13.18 \pm 9.61$ & $24(24)$ \\
\hline Dizziness & $12.23 \pm 11.23$ & $24(26.50)$ \\
\hline Loss of appetite & $14.13 \pm 7.71$ & $24(33)$ \\
\hline
\end{tabular}

their onset and duration as per our study are shown in Figure 3 and Table 3 .

Other symptoms perceived by the participants were weakness of the vaccinated arm (9.1\%), nausea (8\%), flulike symptoms $(7.3 \%)$, swelling or nodule in the injection site $(5.1 \%)$, altered sensation over vaccinated site $(5.1 \%)$, redness of eyes $(2.6 \%)$, vomiting $(2.6 \%)$, burning sensation of eyes (1.8\%), insomnia (1.8\%), loose motion (1.5\%), anxiety $(1.1 \%)$, rashes $(1.1 \%)$, redness on injection site $(1.1 \%)$ and chest pain (1.1\%). Some participants also reported blurring of vision $(0.4 \%)$, muscle cramps $(0.4 \%)$ and excessive sleepiness $(0.4 \%)$ post vaccination.

The findings of simple binary logistic regression of AEFI severity (no and mild Versus Moderate) are shown in Table 4. This gave the inference that Age $(P=0.000)$ and gender $(P=0.001)$ had a significant effect on AEFI severity. Therefore, only the age and gender were forwarded into the multiple logistic regression model while factors like ethnic groups, BMI and alcohol were dropped. The proposed multiple logistic regression model along with estimates of parameters are provided in Table 5 . Gender appears to be a significant factor in determining the severity of AEFI 
Table 4. Effect of possible determinants on severity of AEFI

\begin{tabular}{|l|c|c|}
\hline Factors & Odds ratio (OR) & p-value \\
\hline Ethnic Groups & 0.995 & 0.983 \\
\hline Age & 0.963 & 0.001 \\
\hline Gender & 0.351 & $<0.001$ \\
\hline BMl & 0.998 & 0.929 \\
\hline Alcohol & 0.91 & 0.733 \\
\hline
\end{tabular}

Table 5. Potential determinants of Severity of AEFI given by final Model

\begin{tabular}{|l|l|c|c|c|}
\hline \multicolumn{2}{|l|}{ Variables } & P-value & OR & 95\% confidence interval \\
\hline \multirow{2}{*}{ Gender } & Female & & & \\
\cline { 2 - 2 } & Male $^{*}$ & 0.001 & 2.369 & $1.398-4.014$ \\
\hline Age (in years) & & 0.067 & 0.977 & $0.954-1.002$ \\
\hline
\end{tabular}

" $=$ Reference Group

(OR=2.369, $P=0.001)$. Additionally, females are more than twice likely to develop moderate AEFI as compared to males, keeping age constant. Age, however, is statistically non-significant $(\mathrm{OR}=0.977$ and $P=0.067)$ as per the final model. Omnibus test for model coefficients was significant ( $\chi 2=21.40, P<0.001$ ), implying that the proposed model is better than the null model. Nagelkerke R square is obtained as 0.10 . Moreover, the Hosmer and Lemeshow test was not significant ( $\chi 2=11.756, P=0.162$ ). Hence, we can say that the proposed model is a good fit.

\section{DISCUSSION}

ChAdOx1 nCoV-19 vaccination of the population in a western hilly region of Nepal revealed a high incidence of AEFI. Among them, the "very common" AEFIs perceived were pain on the injection site, generalized weakness, fever, headache, joint and muscle pain, dizziness and loss of appetite. The average onset of these symptoms varied from 9 hours to 14 hours post vaccination. There was a significant variation in the duration of these symptoms.

A systematic review of adverse events following COVID-19 vaccination has suggested that COVID-19 vaccination can lead to mild to moderate reactions. The serious reactions are found unrelated to vaccination. ${ }^{14}$ Our study has also supported these findings. The phase $2 / 3$ trial of the ChAdOx1 nCoV-19 vaccine has shown the rate of post vaccination symptoms to be $86 \%$ in the $18-55$ age group. ${ }^{8}$ A survey from Nepal done on 5591 participants via phone calls after the use of the first dose of the ChAdOx1 nCoV-19 vaccine showed that $85.04 \%$ of the population had AEFI. It also showed that some of the systemic effects of post vaccination were more evident among participants with a history of COVID-19 infection. ${ }^{9}$ An online survey from India which included 5396 people has shown that females are more likely to report post vaccination symptoms and the frequency of

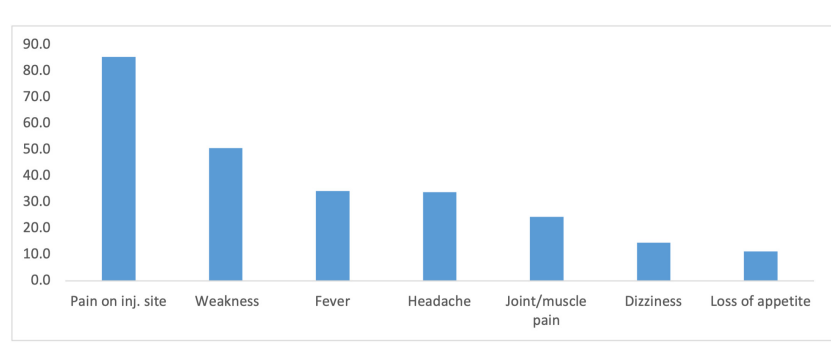

Figure 3. Frequency (\%) of very common AEFI with COVID-19 vaccine

symptoms declines with age. However, the findings of this survey do not suggest any difference in symptoms among those who had COVID-19 infection in the past. ${ }^{10}$ A retrospective study from Korea included 994 health care workers who took the ChAdOx1 $\mathrm{nCoV}-19$ vaccine (where the data was collected using mobile vaccine adverse events reporting system), has also shown that the severity of most AEFIs to be mild-to-moderate and the severity of AEFI to be less in older age group. ${ }^{11}$ Our study has shown that the severity of AEFI was moderate in intensity in the majority of the population. The chances of moderate AEFI were found to vary with age and sex significantly in binary logistic regression. In multiple logistic regression model, the age (in years) was found statistically non-significant. The results from our study are supporting the existing evidences on the variation of AEFI with gender. In other studies the severity of AEFI was found to decrease with age. This was supported by simple binary logistic regression model. The final model in our study is not supporting the existing evidences on variation of AEFI with age. Our study is different from the other studies performed on AEFI with COVID-19 vaccine because it was carried out in a rural setting of a developing country, with a good raport between interviewers 
and the participants. This study has given a model to study the variation of severity of AEFI with different determinants and has quantified the variation of AEFI with gender. The findings from this study may help health experts in formulating plans and policies to improve the vaccine response in the mass vaccination campaigns against COVID-19.

The participants during the second drive of vaccination were administrative officials, bankers, journalists, and those who missed their vaccination during the first drive. Being the active and majority to be educated population, the chances of false-positive and false-negative responses were less. Simple randomization minimized selection bias. As the local level medical practitioners conducted the interview, the response rate to the questionnaire was good, the rapport with the participants was well built, reducing the chances of false reporting. The participants received information from their doctor about receiving a possible phone call by random selection. It could increase the possibility of the participants noting their symptoms more effectively. These were the strengths of our study. This study has tried to explain the onset, duration and severity of symptoms perceived post vaccination. This study included a less number of participants with comorbidities and those with a history of COVID-19 infection. So, the possible effect of comorbidities and history of COVID-19 infection on the severity of AEFI could not be analyzed. We could use only two variables to develop a multiple regression model. There were few participants above 60 years of age. The sample size was not powerful enough to address the rare adverse events. The study included the limited group of people of limited region so the results might not be generalizable. The Nagelkerke R square was calculated to be $10 \%$ which suggested that our predictors explain only $10 \%$ of the variation in the severity of AEFI. We found that the frequency of pain on the injection site, a usual complaint after intramuscular injections, is higher than other systemic adverse events. This adverse event has increased the overall frequency of AEFI. Although the recall bias has been minimized, there might have been some possible recall bias on the onset and duration of the symptoms perceived. These were the limitations of our study.

\section{CONCLUSIONS}

The prevalence of AEFI following vaccination against COVID-19 is found to be high in our study. The majority of the participants perceived moderate-intensity AEFI followed by mild AEFI. There were a fewer number of participants who did not perceive any AEFI. None of the participants perceived severe AEFI. The severity of AEFI is more in females compared to males. Age didn't come out as a predictor for severity of AEFI. Post-vaccination counseling targeting women will improve the acceptance of the vac- cine. There may be some other variables that can determine the severity of AEFI that may have not been included in our study due to the defined professionals included for the scheduled drive of the vaccination. Further studies with larger sample size, preferably nationwide, and broad participation of older age groups, those with comorbidities and individuals who have acquired COVID-19 infection need to be done to find other determinants of AEFI.

\section{ACKNOWLEDGEMENTS}

We acknowledge the support of all staff of Galkot primary health care center, Nepal, District Health office, Baglung, Nepal, National health research council, Nepal and all the study participants who helped us in conducting this study. The study was approved by the Nepal Health Research Council, Nepal (ERB protocol Registration no. 125/2021, Approved on 25 February 2021). We would also like to extend our thanks to Dr. Sudarshan Gautam for helping us in finalization of the manuscript.

\section{FUNDING}

This work did not receive any institutional, private and corporate financial support.

\section{AUTHORSHIP CONTRIBUTION}

Conceptualization and methodology: AG, ND, UB, Acquisition and analysis: AG, ND, SP, BP UB, PS, Interpretation of data for the work: AG, BP, SG,SU,SKS, Drafting the work: AG, PS, UB, SG, ND, SP, BP, SU, SKS, Revising it critically for important intellectual content: SU, SKS, Final approval of the version to be published and agreement to be accountable for all aspects of the work by all of the authors.

\section{COMPETING INTERESTS}

The authors completed the Unified Competing Interest form at www.icmje.org/coi disclosure.pdf (available upon request from the corresponding author), and declare no conflicts of interest.

\section{CORRESPONDENCE TO:}

Arun Gautam

MBBS, PG Scholar

Department of Internal Medicine

BP Koirala Institute of Health Sciences, Dharan, Nepal

Postal code: 56700

arungautam5990@gmail.com 


\section{REFERENCES}

1. Lurie N, Saville M, Hatchett R, Halton J. Developing Covid-19 Vaccines at Pandemic Speed. N Engl J Med. 2020;382(21):1969-1973. doi:10.1056/nejmp2005630

2. Kochhar S, Salmon DA. Planning for COVID-19 vaccines safety surveillance. Vaccine. 2020;38(40):6194-6198. doi:10.1016/j.vaccine.2020.0 $\underline{7.013}$

3. Sah R, Shrestha S, Mehta R, et al. AZD1222 (Covishield) vaccination for COVID-19: Experiences, challenges, and solutions in Nepal. Travel Med Infect Dis. 2021;40:101989. doi:10.1016/i.tmaid.2021.10198 $\underline{9}$

4. Bhattarai S, Dhungana J. A million-dose success for Nepal: insights from Nepal's national vaccine deployment plan against COVID-19. J Travel Med. 2021;28(4):taab027. doi:10.1093/itm/taab027

5. Poudel A. Nepal begins first phase of COVID-19 vaccination drive. The Kathmandu Post. https://kathm andupost.com/health/2021/01/27/nepal-begins-firstphase-of-vaccination-drive-against-covid-19. Published January 28, 2021. Accessed May 23, 2021.

6. Total coronavirus cases in Nepal. Worldometer. Published June 26, 2021. Accessed June 26, 2021. http s://www.worldometers.info/coronavirus/country/nepa $\underline{1}$

7. Covid-19 dashboard. Ministry of Health and Population Nepal. Published June 26, 2021. Accessed June 26, 2021. https://covid19.mohp.gov.np/\#/

8. Ramasamy MN, Minassian AM, Ewer KJ, et al. Safety and immunogenicity of ChAdOx1 nCoV-19 vaccine administered in a prime-boost regimen in young and old adults (COV002): a single-blind, randomised, controlled, phase $2 / 3$ trial. Lancet. 2020;396(10267):1979-1993. doi:10.1016/s0140-673 6(20)32466-1
9. Shrestha S, Devbhandari RP, Shrestha A, et al. Adverse events following the first dose of ChAdOx1 nCoV-19 (COVISHIELD) vaccine in the first phase of vaccine roll out in Nepal. J Patan Acad Health Sci. 2021;8(1):9-17. doi:10.3126/jpahs.v8i1.36242

10. Jayadevan R, Shenoy RS, Anithadevi TS. Survey of symptoms following COVID-19 vaccination in India. medRxiv. Published online January 1, 2021. doi:10.110 $1 / 2021.02 .08 .21251366$

11. Jeon M, Kim J, Oh CE, Lee JY. Adverse Events Following Immunization Associated with Coronavirus Disease 2019 Vaccination Reported in the Mobile Vaccine Adverse Events Reporting System. J Korean Med Sci. 2021;36(17):e114. doi:10.3346/jkms.2021.3 6.e114

12. National Cancer Institute (US). Cancer Therapy Evaluation Program. Common Terminology Criteria for Adverse Events: (CTCAE). Cancer Therapy Evaluation Program; 2003.

13. Tozzi AE, Asturias EJ, Balakrishnan MR, Halsey NA, Law B, Zuber PLF. Assessment of causality of individual adverse events following immunization (AEFI): a WHO tool for global use. Vaccine. 2013;31(44):5041-5046. doi:10.1016/i.vaccine.2013.0 $\underline{8.087}$

14. Kaur RJ, Dutta S, Bhardwaj P, et al. Adverse Events Reported From COVID-19 Vaccine Trials: A Systematic Review. Ind J Clin Biochem. Published online 2021:1-13. doi:10.1007/s12291-021-00968-z 